\title{
Temporopolar amygdalohippocampectomy: seizure control and postoperative outcomes
}

\author{
João Paulo Sant Ana Santos de Souza, MD, ${ }^{1}$ Gabriel Ayub, MD, ${ }^{4}$ Mateus Nogueira, PhD, ${ }^{1}$ \\ Tamires Zanao, MSc, ${ }^{1}$ Tátila Martins Lopes, PhD, ${ }^{1}$ Luciana Ramalho Pimentel-Silva, PhD, ${ }^{1}$ \\ Vinicius Domene, MD, ${ }^{1}$ Gabriel Marquez, MD, ${ }^{1}$ Clarissa Lin Yasuda, MD, PhD, ${ }^{1,3}$ \\ Letícia Franceschet Ribeiro, MD, ${ }^{1}$ Brunno M. Campos, PhD, ${ }^{1}$ José Vasconcellos, MD, PhD, ${ }^{4}$ \\ Fabio Rogerio, MD, PhD, ${ }^{5}$ Andrei Fernandes Joaquim, MD, PhD, ${ }^{2}$ Fernando Cendes, MD, PhD, ${ }^{1,3}$ \\ Helder Tedeschi, MD, PhD, ${ }^{2}$ and Enrico Ghizoni, MD, PhD ${ }^{1,2}$
}

${ }^{1}$ Neuroimaging Laboratory (LNI), Department of Neurology, Divisions of ${ }^{2}$ Neurosurgery and ${ }^{3} \mathrm{Clinical}$ Neurology, ${ }^{4}$ Department of Ophthalmology, and ${ }^{5}$ Department of Anatomical Pathology, University of Campinas (UNICAMP), Campinas, São Paulo, Brazil

OBJECTIVE The objective of this study was to evaluate the efficacy and safety of a modified surgical approach for the treatment of temporal lobe epilepsy secondary to hippocampal sclerosis (HS). This modified approach, called temporopolar amygdalohippocampectomy (TP-AH), includes a transsylvian resection of the temporal pole and subsequent amygdalohippocampectomy utilizing the limen insula as an anatomical landmark.

METHODS A total of 61 patients who were diagnosed with HS and underwent TP-AH between 2013 and 2017 were enrolled. Patients performed pre- and postoperative diffusion tensor imaging and were classified according to Engel's scale for seizure control. To evaluate the functional preservation of the temporal stem white-matter fiber tracts, the authors analyzed postoperative Humphrey perimetries and pre- and postoperative neurocognitive performance (Rey Auditory Verbal Learning Test [RAVLT], Weschler Memory Scale-Revised [WMS-R], intelligence quotient [IQ], Boston Naming Test [BNT], and semantic and phonemic fluency). Demographic data and surgical complications were also recorded and described.

RESULTS After a median follow-up of $36 \pm 16$ months, 46 patients (75.4\%) achieved Engel class I, of whom 37 (60.6\%) were Engel class IA. No significant changes in either the inferior frontooccipital fasciculus and optic radiation tractography were observed postoperatively for both left- and right-side surgeries. Reliable perimetry was obtained in 40 patients $(65.6 \%)$, of whom $27(67.5 \%)$ did not present any visual field defects (VFDs) attributable to surgery, while 12 patients (30\%) presented with quadrant VFD, and 1 patient (2.5\%) presented with hemifield VFD. Despite a significant decline in verbal memory ( $p=0.007$ for WMS-R, $p=0.02$ for RAVLT recognition), there were significant improvements in both $I Q(p<0.001)$ and visual memory $(p=0.007)$. Semantic and phonemic fluency, and scores on the BNT, did not change postoperatively.

CONCLUSIONS TP-AH provided seizure control similar to historical temporal lobe approaches, with a tendency to preserve the temporal stem and a satisfactory incidence of VFD. Despite a significant decline in verbal memory, there were significant improvements in both $I Q$ and visual memory, along with preservation of executive function. This approach can be considered a natural evolution of the selective transsylvian approach.

https://thejns.org/doi/abs/10.3171/2020.3.JNS192624

KEYWORDS amygdalohippocampectomy; temporal lobe epilepsy; hippocampal sclerosis; tractography; temporal stem

ABBREVIATIONS AD = axial diffusivity; $A T L=$ anterior temporal lobectomy; $B N T$ = Boston Naming Test; $D T I=$ diffusion tensor imaging; EZ = epileptogenic zone; FA = fractional anisotropy; $\mathrm{HS}=$ hippocampal sclerosis; IFOF = inferior frontooccipital fasciculus; $I Q=$ intelligence quotient; $M C A=$ middle cerebral artery; $M D=$ mean diffusivity; MTLE = mesial TLE; OR = optic radiation; RAVLT = Rey Auditory Verbal Learning Test; RD = radial diffusivity; TLE = temporal lobe epilepsy; TP-AH = temporopolar amygdalohippocampectomy; TS = temporal stem; UF = uncinate fasciculus; VFD = visual field defect; WAIS-R = Weschler Adult Intelligence Scale-Revised; WMS-R = Weschler Memory Scale-Revised.

SUBMITTED September 27, 2019. ACCEPTED March 10, 2020.

INCLUDE WHEN CITING Published online May 15, 2020; DOI: 10.3171/2020.3.JNS192624. 
$\mathrm{H}$ IPPOCAMPAL sclerosis (HS) corresponds to the main etiology of mesial temporal lobe epilepsy (MTLE) among adults; approximately $30 \%$ of patients are referred to surgery due to pharmacological resistance. ${ }^{1,2}$ The ultimate goal of surgery is resection of the entire epileptogenic zone (EZ) for seizure-freedom outcomes associated with the lowest rate of complications.

In many epilepsy centers around the world, the selective transsylvian approach for amygdalohippocampectomy $(\mathrm{AH})$ is the current standard of care for treating refractory MTLE, and this approach was the standard of care provided by our facility from 2002 to 2012. ${ }^{3}$ Although use of the selective transsylvian approach is intended to preserve the lateral temporal neocortex, postoperative imaging analyses of our patients revealed a frequent temporal pole (TP) atrophy, likely attributable to that surgical approach, as well as a high incidence of temporal stem (TS) damage. ${ }^{4}$ Additionally, meta-analyses and systematic reviews report significant improvement in seizure control, or at least a tendency toward better outcomes, following anterior temporal lobectomy (ATL) compared to selective approaches. ${ }^{5-8}$ This critical information, coupled with our own experience, supported utilizing an anterior opening of the Sylvian fissure to remove the TP, with the limen insula as the posterior limit, to gain better exposure of the uncus and the anterior portion of the temporal horn. We called this approach temporopolar amygdalohippocampectomy (TP-AH).

The present study sought to evaluate the efficacy and safety of TP-AH for refractory MTLE secondary to HS. Our primary objective was to demonstrate that this novel technique provides good seizure control and an acceptable profile of complications by comparing pre- and postoperative variables of patients who underwent TP-AH.

\section{Methods}

\section{Patients}

A prospective observational cohort study was performed with the first 61 consecutive patients with medically refractory epilepsy secondary to HS who underwent TP-AH at the Epilepsy Service of the University of Campinas (between 2013 and 2017) and completed at least 1 year of follow-up. Patients with any additional progressive disease and/or previous epilepsy surgery were excluded. The present study was approved by the Ethics Committee of our institution. All patients who were included in the study signed an informed consent form.

\section{Preoperative Evaluation}

All patients underwent standard preoperative evaluation at our outpatient clinic, which included: clinical assessment, scalp EEG showing evidence of unilateral epileptogenic discharge arising from the anteromesial temporal region, refractoriness to at least two antiepileptic drug regimens, neuropsychological assessment, and 3T-MRI evidence of hippocampal atrophy (T1-, T2- and FLAIR-weighted images). Continuous long-term video-EEG was performed in 34 patients. ${ }^{9}$ Cases were discussed individually by a multidisciplinary team, including neurosurgeons, neurologists, epileptologists, and neuroradiologists before surgery.
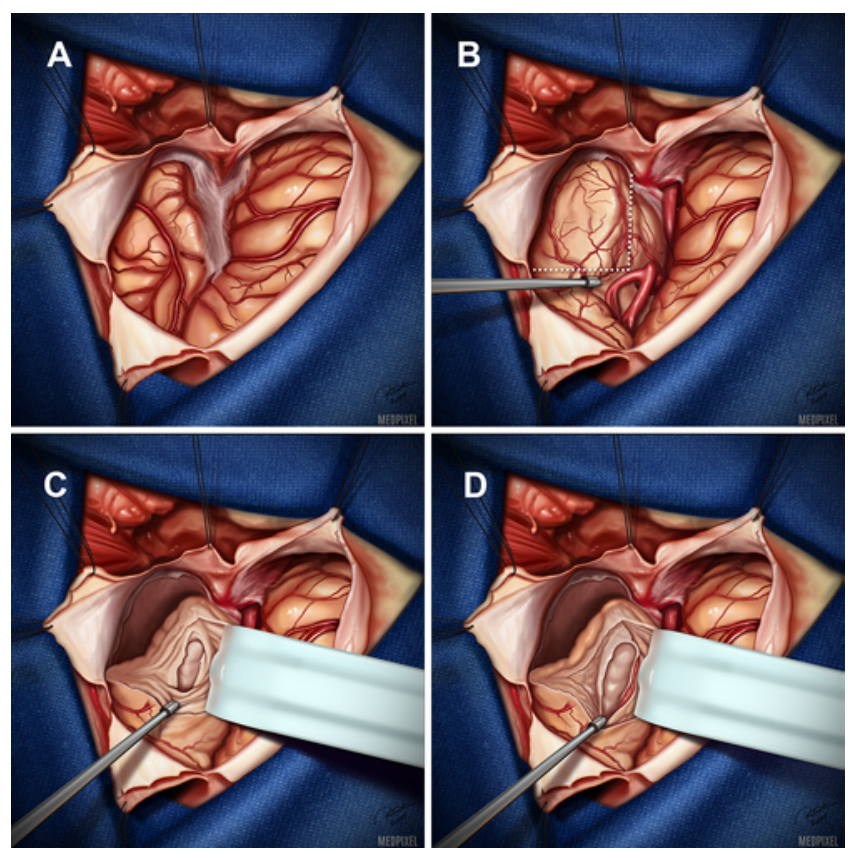

FIG. 1. Schematic TP-AH after left-sided pretemporal exposure. A: Sylvian fissure before dissection. B: After Sylvian fissure dissection, an incision (dotted line) is performed from the anteromesial aspect of the rhinal sulcus to the limen insula, and then directed $90^{\circ}$ toward the lateral temporal lobe surface. C: The TP has been removed and the temporal horn is approached. D: The temporal horn is opened, and the hippocampus and choroidal plexus are exposed. Copyright João Paulo Sant Ana Santos de Souza. Published with permission. Figure is available in color online only.

\section{Surgical Approach}

The modified surgical technique addressed in this study was performed as described in a previous paper. ${ }^{10}$ The procedure began with a pretemporal craniotomy, followed by a limited Sylvian fissure dissection (sphenoidal portion and initial opercular portion) to expose the internal carotid artery, middle cerebral artery (MCA) M1 segment, MCA bifurcation, uncus, and limen insula (Fig. 1, Supplemental Fig. 1). After all of these critical landmarks were sufficiently exposed, we made an incision running from the anteromedial impression of the rhinal sulcus, separating the TP from the uncus, up to the limen insula, and then the incision was directed $90^{\circ}$ to the lateral surface of the temporal lobe, thus limiting the extension of TP resection. After resection of the TP was accomplished, the uncus was fully exposed, allowing for an anteroposterior approach to the temporal horn of the lateral ventricle thorough its anterior wall, the amygdala. This approach created sufficient space to facilitate removal of the hippocampus and parahippocampus en bloc, so that histopathological analysis could be performed. The lateral geniculate body served as the posterior limit of the hippocampal resection and the collateral eminence served as its lateral limit. Finally, the amygdala was examined and its resection completed, with the optic tract serving as the superior and medial limits.

Utilizing TP-AH as our surgical approach enabled us to provide distinct and well-preserved specimens of each patient's TP, body of hippocampus, and amygdala. 


\section{Postoperative Evaluation}

A histopathological evaluation was performed by an

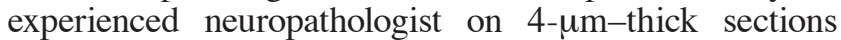
from formalin-fixed, paraffin-embedded specimens and stained with H \& E. Specifically, HS was characterized by pyramidal cell loss and gliosis. ${ }^{11}$ The seizure control rate was assessed using Engel's classification, collected during outpatient clinic follow-up. Three-Tesla MRI was acquired before discharge and at least 2 months after surgery. Herein, an experienced neuroradiologist analyzed T1-, T2-, FLAIR- and diffusion-weighted images. Diffusion tensor imaging (DTI) acquisition was performed with no less than 2 months of follow-up.

\section{Perimetry}

Before perimetry testing (24-2 Humphrey digital examination), all patients underwent best-corrected visual acuity tests to exclude comorbidities and refraction errors that could confer bias. Results were evaluated by a senior ophthalmologist (J.V.). Applied exclusion criteria were: fixation loss $\geq 20 \%$, false positive $\geq 33 \%$, false negative $\geq$ $33 \%$, "clover leaf" pattern, any previous ophthalmological comorbidity that could interfere with the result of perimetry, and refusal to participate in the study.

\section{Diffusion Tensor Imaging}

DTI acquisition and postprocessing, as well as optic radiation (OR) tractography, were used in accordance with a protocol previously published by our group. ${ }^{12}$ We obtained the total number of identifiable streamlines for each tract pre- and postoperatively (counted numerically as an integer number; each fiber with a length between 10-500 mm was considered as a single streamline), as well as microstructural variables (fractional anisotropy [FA], mean diffusivity [MD], axial diffusivity [AD], and radial diffusivity $[R D]$, each parameter varying from 0 to 1$)$. The tracking strategy used for the inferior frontooccipital fasciculus (IFOF) is presented in Supplemental Protocol 1.

\section{Neuropsychological Assessment}

We performed a neuropsychological evaluation before surgery in all patients and postoperatively in 23 patients. Verbal and nonverbal memories were evaluated with the Weschler Memory Scale-Revised (WMS-R) and the Rey Auditory Verbal Learning Test (RAVLT). We calculated the intelligence quotient (IQ) using block design and vocabulary subtests (Weschler Adult Intelligence Scale-Revised [WAIS-R]). Executive function was evaluated using semantic and phonemic fluency and the Boston Naming Test (BNT). Except for the IQ test, all test results were derived from normative data obtained from members of the Brazilian population. ${ }^{13}$

\section{Statistical Analysis}

Statistical analysis was performed with Statistical Analysis System for Windows software (version 9.4, SAS Institute Inc.) and SPSS (version 24, IBM Corp.). ANOVA for repeated measures was used to compare DTI and neuropsychological variables between pre- and postoperative evaluations. This model was adjusted according to 1) the patient's age at surgery, and 2) the time interval between surgery and postoperative image acquisition. For neuropsychological assessment, we built three different multivariate repeated measures ANOVAs for the following domains: memory (RAVLT and WMS-R), IQ (WAIS-R), and executive function (semantic and phonemic fluency, and the BNT). The three models were adjusted to the interval between surgery and postoperative evaluation. We also ran the same models on neuropsychological data, taking into account the side of surgery (left vs right), brain lateralization (dominant vs nondominant), assessed Engel outcomes (Engel class IA vs other classes), and length of the term of interaction between pre- and postoperative evaluations. Data and error distribution were evaluated using the Shapiro-Wilk test and quantile-quantile plots. Overall Engel classifications, imaging findings, and perimetric outcomes were evaluated semiquantitatively. A $p$ value $<0.05$ was considered statistically significant for all comparisons. The false discovery rate was applied to correction for multiple comparisons.

\section{Results \\ Demographic Features}

A total of 61 patients with a mean age at surgery of 40.9 \pm 13.0 years who underwent TP-AH (33 left side, 28 right side) were included in the present study. Additional demographic features are summarized in Table 1.

\section{Histopathological Findings}

HS was confirmed in all cases, with the exception of 1 patient. In this case, neuronal loss was not observed in either H \& E- or NeuN-stained sections, whereas immunohistochemistry for glial fibrillary acidic protein highlighted gliosis in sectors CA1 and CA4.

Concerning the TP, gliosis at the molecular cortical layer was the most common finding, observed in 58 (95\%) of 61, followed by gliosis with corpora amylacea irregularly distributed at the cortex in $28(45 \%)$, white matter gliosis in 9 (14\%), focal cortical dysplasia (abnormal radial lamination with no dysmorphic neurons or balloon cells) in $2(3.3 \%)$, and heterotopia in $1(1.6 \%)$.

\section{Seizure Control}

After a median follow-up of 36 months (range 1-6.7 years, $64 \%$ of cases with at least 2 years), $46(75 \%)$ of 61 patients were classified as having good seizure control (Engel class I). At the last outpatient clinic visit, 37 patients (61\%) were classified as Engel class IA, $6(9.8 \%)$ as Engel class IB, $1(1.6 \%)$ as Engel class IC, $3(3.3 \%)$ as Engel class ID, $10(16 \%)$ as Engel class II, and 5 patients $(8.2 \%)$ as Engel class III. None of our patients were classified as Engel class IV.

\section{Surgical Complications}

Other than expected visual complications, there were no cases of death or major complications resulting in permanent morbidities. MRI findings that did not result in any clinical repercussions are reported in Supplemental Table 1. 
TABLE 1. Demographics of patients who underwent TP-AH ( $n=61)$

\begin{tabular}{lc}
\hline \multicolumn{1}{c}{ Characteristic } & Value \\
\hline Sex, $\mathrm{n}(\%)$ & $22(36)$ \\
\hline Male & $39(64)$ \\
\hline Female & $10.2 \pm 9.3$ \\
\hline Mean age at seizure onset $\pm \mathrm{SD}, \mathrm{yrs}$ & $40.9 \pm 13.0$ \\
\hline Mean age at surgery \pm SD, yrs & $31.2 \pm 14.4$ \\
\hline Mean duration of epilepsy until surgery \pm SD, yrs & \\
\hline Side of surgery, $\mathrm{n}(\%)$ & $33(54)$ \\
\hline Lt & $28(46)$ \\
\hline Rt & $37.0 \pm 16.1$ \\
\hline Mean follow-up \pm SD, mos & \\
\hline Mean interval \pm SD, mos & $17.7 \pm 14.3$ \\
\hline Between preop DTI \& surgery & $19.9 \pm 15.3$ \\
\hline Between postop DTI \& surgery & $30.1 \pm 19.1$ \\
\hline Between surgery \& perimetry $\dagger$ & 4 \\
\hline Median discharge time, days & \\
\hline
\end{tabular}

${ }^{*}$ Follow-up = the interval between surgery and the last outpatient clinic visit for Engel classification.

† Only patients who fulfilled the inclusion criteria for perimetry were included in this calculation.

$\ddagger$ Interval of time between surgery and the day in which the patient could be discharged from the hospital.

In the series, 6 (9.8\%) of 61 patients experienced transient surgical complications. One patient had an epidural empyema that completely resolved following a 23-day course of vancomycin; 1 patient presented with aseptic meningitis and low fever only, but no bacteria isolated; 1 patient developed hydrocephalus and required a ventriculoperitoneal shunt; 1 patient presented with a CSF leak, under the scalp only, with complete resolution following an external lumbar drain; 1 patient developed a transitory third cranial nerve palsy, but fully recovered, and 1 patient presented with transitory hemiparesis, but fully recovered.

\section{VFDs}

Reliable perimetry was achieved in $40(75 \%)$ of 53 patients, 27 of whom showed no visual field defects (VFDs) attributable to postoperative injury (Fig. 2). VFDs due to TP-AH were restricted to quadrant loss in $12(30 \%)$ of 40 patients, and only 1 patient $(2.5 \%)$ had a hemifield defect.

\section{Tractography Outcomes}

A total of 55 patients underwent both pre- and postoperative DTI. From these patients, it was not possible to track the IFOF in 1 patient and the OR in 4 patients postoperatively. As expected, the uncinate fascicles (UFs) were interrupted in all patients. The analyses of both IFOF and OR tractographies did not reveal significant differences between pre- and postoperative groups in any of the variables analyzed, regardless of the statistical model adopted (Table 2). Final DTI images are shown in Fig. 3.

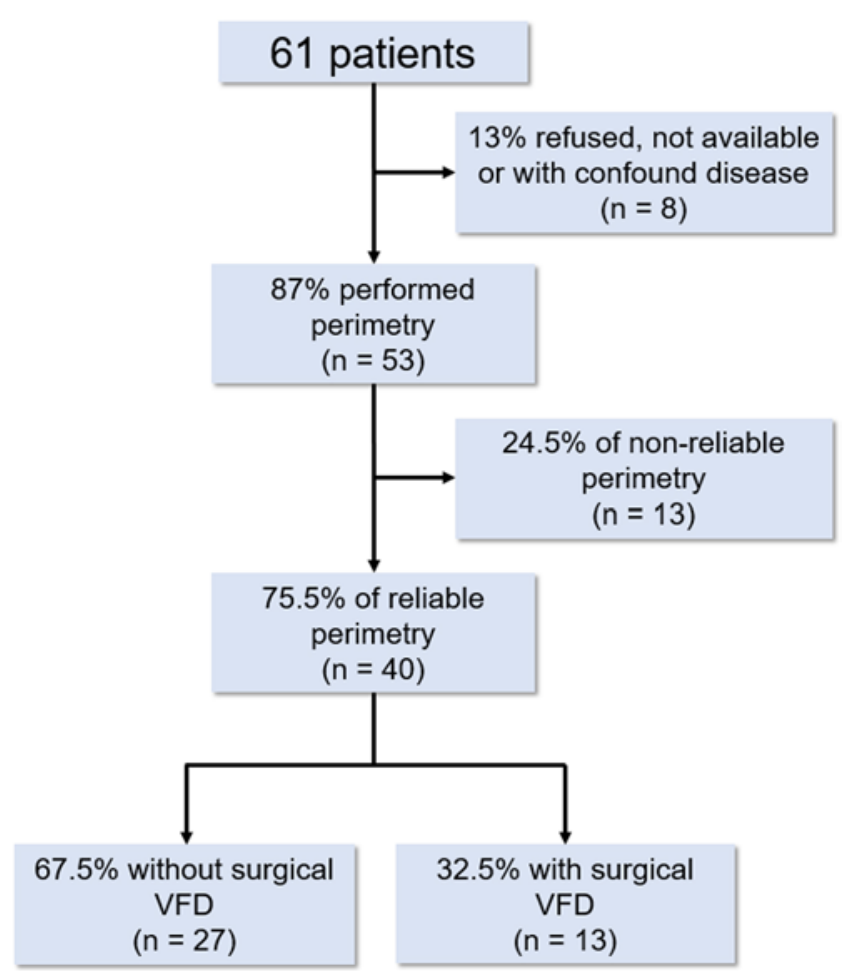

FIG. 2. Perimetry flow diagram. This flow chart shows the number of patients who performed postoperative perimetry. Figure is available in color online only.

\section{Neuropsychological Outcomes}

Twenty-three (38\%) of 61 patients underwent a postoperative evaluation, after a mean postoperative interval of $4.1 \pm 1.2$ years (12 left-sided surgery, 11 right-sided). One patient did not complete preoperative BNT, semantic fluency, and phonemic fluency evaluations, and 1 patient did not complete a postoperative phonemic fluency.

We found significant changes when comparing pre- and postoperative memory evaluations $\left(\mathrm{F}_{6,14}=4.87, \mathrm{p}=0.007\right.$, $\left.\eta_{p}^{2}=0.676\right)$, which included significant improvement in visual memory, but significant declines in verbal memory and recognition. Although no significant changes were observed between pre- and postoperative executive function evaluations $\left(\mathrm{F}_{3,16}=2.02, \mathrm{p}=0.15, \eta_{p}^{2}=0.275\right)$, all of the executive function tests revealed a tendency for postoperative improvement (Table 3). Additionally, significant improvement in IQ was shown when comparing pre- and postoperative evaluations $\left(\mathrm{F}_{1,20}=23.11, \mathrm{p}<0.001, \eta_{p}^{2}=0.536\right)$.

In terms of group analysis, there were no significant effects attributable to the surgery side (all $\mathrm{p}$ values $>0.2$ for executive function, all $\mathrm{p}$ values $>0.2$ for memory, and $\mathrm{p}=$ 0.8 for IQ), brain lateralization (all $\mathrm{p}$ values $>0.1$ for executive function, all $\mathrm{p}$ values $>0.18$ for memory, and $\mathrm{p}=0.9$ for IQ) or Engel outcome (all $p$ values $>0.3$ for executive function, all $\mathrm{p}$ values $>0.4$ for memory, and $\mathrm{p}=0.8$ for IQ).

\section{Discussion}

\section{Technique Development}

The selective transsylvian approach for amygdalohip- 
TABLE 2. Comparisons between pre- and postoperative tractographies

\begin{tabular}{|c|c|c|c|c|c|c|c|}
\hline Side of Surgery/DTI Variable & Mean & SD & Min & Median & $\operatorname{Max}$ & p Value* & $p$ Value \\
\hline \multicolumn{8}{|l|}{ IFOF tract } \\
\hline $\operatorname{Rt}(n=23)$ & & & & & & 0.10 & 0.78 \\
\hline Preop FA & 0.4954 & 0.0295 & 0.4531 & 0.4972 & 0.5718 & & \\
\hline Postop FA & 0.4829 & 0.0306 & 0.4366 & 0.4734 & 0.5601 & & \\
\hline $\mathrm{Lt}(\mathrm{n}=31)$ & & & & & & 0.09 & 0.42 \\
\hline Preop FA & 0.4925 & 0.0250 & 0.4341 & 0.4991 & 0.5327 & & \\
\hline Postop FA & 0.4795 & 0.0243 & 0.4274 & 0.4801 & 0.5221 & & \\
\hline $\mathrm{Rt}$ & & & & & & 0.42 & 0.66 \\
\hline Preop MD & 0.000786 & 0.000036 & 0.000690 & 0.000787 & 0.000844 & & \\
\hline Postop MD & 0.000791 & 0.000033 & 0.000697 & 0.000790 & 0.000839 & & \\
\hline $\mathrm{Lt}$ & & & & & & 0.80 & 0.46 \\
\hline Preop MD & 0.000799 & 0.000035 & 0.000746 & 0.000793 & 0.000866 & & \\
\hline Postop MD & 0.000811 & 0.000045 & 0.000745 & 0.000800 & 0.000924 & & \\
\hline $\mathrm{Rt}$ & & & & & & 0.45 & 0.19 \\
\hline Preop AD & 0.00126 & 0.000051 & 0.00116 & 0.00126 & 0.00137 & & \\
\hline Postop AD & 0.00125 & 0.000053 & 0.00114 & 0.00126 & 0.00133 & & \\
\hline $\mathrm{Lt}$ & & & & & & 0.33 & 0.56 \\
\hline Preop AD & 0.00128 & 0.000043 & 0.00118 & 0.00128 & 0.00136 & & \\
\hline Postop AD & 0.00128 & 0.000055 & 0.00119 & 0.00129 & 0.00141 & & \\
\hline $\mathrm{Rt}$ & & & & & & 0.18 & 0.60 \\
\hline Preop RD & 0.000548 & 0.000037 & 0.000441 & 0.000549 & 0.000612 & & \\
\hline Postop RD & 0.000559 & 0.000036 & 0.000470 & 0.000560 & 0.000604 & & \\
\hline $\mathrm{Lt}$ & & & & & & 0.15 & 0.86 \\
\hline Preop RD & 0.000557 & 0.000037 & 0.000505 & 0.000549 & 0.000639 & & \\
\hline Postop RD & 0.000575 & 0.000045 & 0.000503 & 0.000558 & 0.000681 & & \\
\hline $\mathrm{Rt}$ & & & & & & 0.61 & \\
\hline Preop NF & 3277.8 & 1633.6 & 1197.0 & 2971.0 & 8090.0 & & 0.67 \\
\hline Postop NF & 3024.7 & 1853.0 & 7.0 & 2575.0 & 6643.0 & & \\
\hline $\mathrm{Lt}$ & & & & & & 0.81 & 0.22 \\
\hline Preop NF & 2736.5 & 1019.3 & 523.0 & 2615.0 & 4998.0 & & \\
\hline Postop NF & 2737.5 & 1157.0 & 690.0 & 2994.0 & 5032.0 & & \\
\hline \multicolumn{8}{|l|}{ OR tract } \\
\hline $\operatorname{Rt}(n=21)$ & & & & & & 0.62 & 0.36 \\
\hline Preop FA & 0.4879 & 0.0317 & 0.4297 & 0.4879 & 0.5815 & & \\
\hline Postop FA & 0.4869 & 0.0275 & 0.4231 & 0.4880 & 0.5331 & & \\
\hline Lt $(n=30)$ & & & & & & 0.47 & 0.85 \\
\hline Preop FA & 0.4829 & 0.0331 & 0.4161 & 0.4872 & 0.5581 & & \\
\hline Postop FA & 0.4826 & 0.0417 & 0.3441 & 0.4931 & 0.5393 & & \\
\hline Rt & & & & & & 0.12 & 0.94 \\
\hline Preop MD & 0.000800 & 0.000046 & 0.000669 & 0.000792 & 0.000879 & & \\
\hline Postop MD & 0.000825 & 0.000049 & 0.000741 & 0.000812 & 0.000930 & & \\
\hline $\mathrm{Lt}$ & & & & & & 0.64 & 0.98 \\
\hline Preop MD & 0.000814 & 0.000049 & 0.000742 & 0.000806 & 0.000928 & & \\
\hline Postop MD & 0.000820 & 0.000052 & 0.000747 & 0.000809 & 0.000930 & & \\
\hline Rt & & & & & & 0.72 & 0.22 \\
\hline Preop AD & 0.00127 & 0.000063 & 0.00117 & 0.00126 & 0.00140 & & \\
\hline Postop AD & 0.00131 & 0.000082 & 0.00119 & 0.00130 & 0.00146 & & \\
\hline
\end{tabular}


» CONTINUED FROM PAGE 1048

TABLE 2. Comparisons between pre- and postoperative tractographies

\begin{tabular}{|c|c|c|c|c|c|c|c|}
\hline Side of Surgery/DTI Variable & Mean & SD & Min & Median & Max & p Value* & p Value† \\
\hline \multicolumn{8}{|l|}{ OR tract (continued) } \\
\hline $\mathrm{Lt}$ & & & & & & 0.86 & 0.56 \\
\hline Preop AD & 0.00129 & 0.000077 & 0.00117 & 0.00129 & 0.00151 & & \\
\hline Postop AD & 0.00130 & 0.000069 & 0.00119 & 0.00130 & 0.00145 & & \\
\hline Rt & & & & & & 0.36 & 0.86 \\
\hline Preop RD & 0.000564 & 0.000046 & 0.000418 & 0.000571 & 0.000642 & & \\
\hline Postop RD & 0.000582 & 0.000041 & 0.000501 & 0.000581 & 0.000679 & & \\
\hline $\mathrm{Lt}$ & & & & & & 0.35 & 0.29 \\
\hline Preop RD & 0.000577 & 0.000046 & 0.000518 & 0.000567 & 0.000677 & & \\
\hline Postop RD & 0.000582 & 0.000057 & 0.000509 & 0.000563 & 0.000730 & & \\
\hline Rt & & & & & & 0.49 & 0.18 \\
\hline Preop NF & 257.0 & 250.1 & 13.0 & 213.0 & 944.0 & & \\
\hline Postop NF & 183.8 & 163.3 & 2.0 & 161.0 & 492.0 & & \\
\hline $\mathrm{Lt}$ & & & & & & 0.85 & 0.053 \\
\hline Preop NF & 432.5 & 342.2 & 6.0 & 374.0 & 1307.0 & & \\
\hline Postop NF & 382.5 & 467.5 & 3.0 & 195.5 & 1681.0 & & \\
\hline
\end{tabular}

$\mathrm{NF}=$ number of fibers.

${ }^{*}$ Adjusted to patient's age at surgery.

$\dagger$ Adjusted to the time interval between surgery and image acquisition.

pocampectomy remained the standard of care for MTLE until 2012 at our facility. ${ }^{3}$

Our neuroimaging team observed during the late postoperative imaging analysis that almost all patients suffered TP atrophy accompanied by high relaxometry signal intensity, ${ }^{4}$ which is suggestive of gliosis and a possible loss of function. Concerning TP function, the participation of this structure in cognitive performance is a matter of debate. It appears that the TP plays a role in semantic memory, but the literature is scarce on the issue..$^{14}$ This hypothesis was formed due to the bilateral lesions of the TP in semantic dementia, encephalitis caused by herpes simplex, or Alzheimer's disease. ${ }^{14,15}$ However, little is known about unilateral injury restricted to the TP. Tsapikini et al. compared a group of 20 patients with acute stroke in TP with 28 patients without TP damage matched for infarct volume. Comparisons between the groups showed no difference in either auditory comprehension or naming tasks. ${ }^{16}$ Lambon Ralph et al. also found no difference in a battery of tests sensitive to semantic impairment when they compared patients with unilateral anterior temporal damage (following vascular injury or resection for tumor or epilepsy) and controls. ${ }^{15}$ This latter study reinforced that bilateral damage to the anterior temporal lobe is required to cause semantic impairment.

As the occurrence of TP atrophy was consistently observed in our results, we accordingly changed our surgical protocol to a transsylvian route to expose the uncus, and removed the TP, using the limen insula as the posterior limit, and approached the temporal horn through its anterior wall (amygdala). We called this approach temporopolar amygdalohippocampectomy, and as we showed, it was a natural evolution of the original transsylvian amygdalo- hippocampectomy. This surgical approach is intended to minimize the extent of resection of the temporal lobe neocortex, and thereby preserve as much of the TS as possible.

\section{Seizure Control}

Meta-analyses and systematic reviews indicate a significant improvement in seizure control or at least a tendency toward better outcomes following ATL compared to selective approaches. ${ }^{5-8}$ The Engel class I rate was found to be $32 \%$ higher for ATL when compared to the selective approach.7 Consistent with this data, Elliott et al. demonstrated ATL to be superior to selective approaches also in pediatric patients ( $77 \%$ vs $44 \%$ Engel class I rate). ${ }^{17}$ In contrast, pro-selective groups advocate in favor of restricted resection to reduce surgical morbidity. ${ }^{18-20}$ It is intuitive to think that restricted resection leads to less structural damage, but in the case of selective approaches, this fact is far from being validated. ${ }^{21,22}$ Restricted resection is also a major concern if we consider that the EZ is not always confined to the amygdala and the hippocampus. In a series of 168 patients with TLE associated with normal MRI or imaging signs of HS, Barba et al. demonstrated that "temporal plus epilepsy" is a major determinant of temporal lobe surgery failures. ${ }^{23}$ Indeed, the risk of resective surgical failure was 5.06 times greater in patients with temporal plus epilepsy than in those with typical MTLE.

Chabardès et al..$^{24}$ assessed 48 patients with TLE who underwent stereoelectroencephalography monitoring of TP and tailored ATL. In 23 patients (48\%, group I), the TP was involved at the onset of the seizure, before, or concurrently with the hippocampus. In the remaining 25 patients (group II), the TP was involved 16.4 seconds after the hip- 

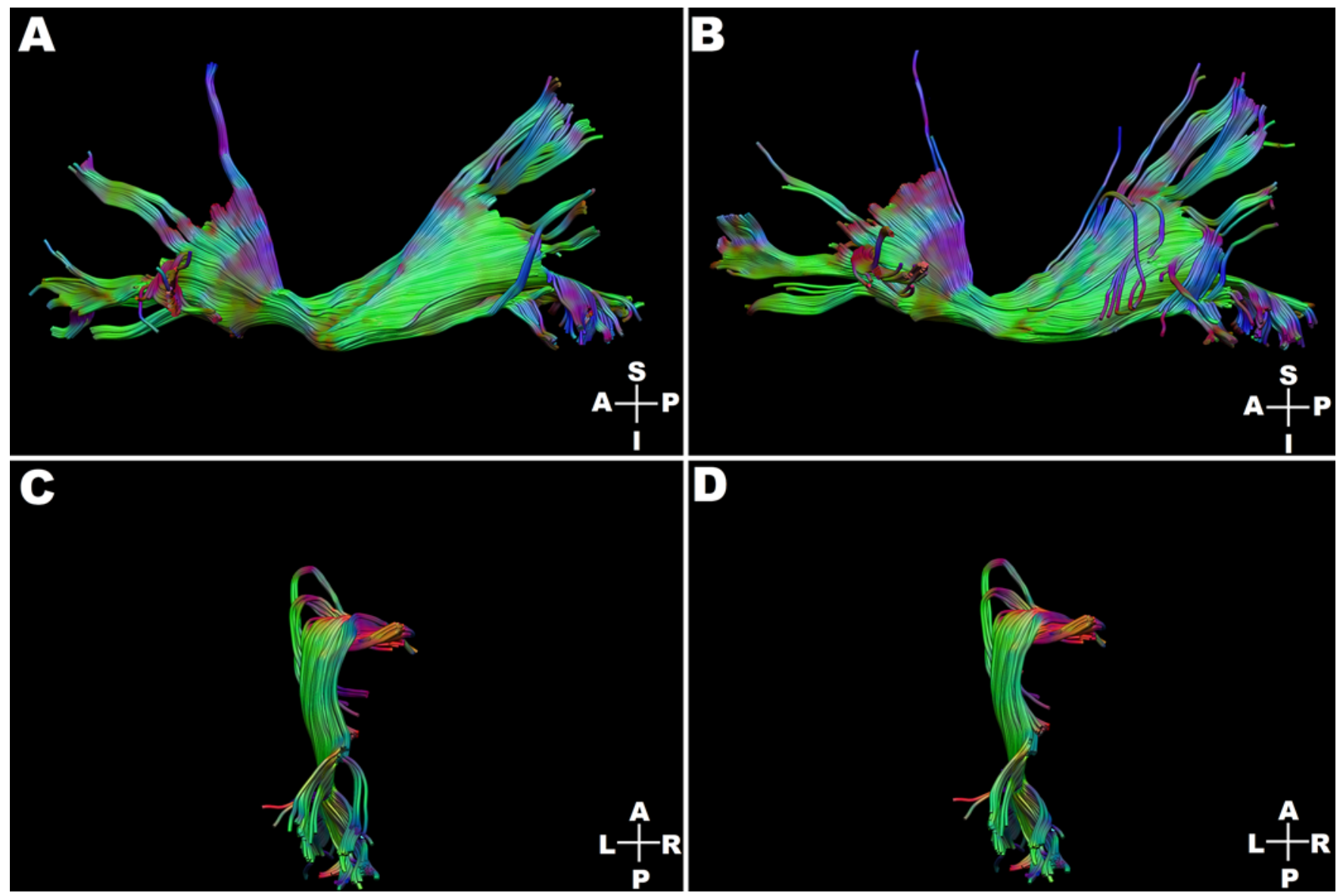

FIG. 3. Tractography final images. A: Lateral view of a left preoperative IFOF. B: Lateral view of the postoperative IFOF from the same patient shown in panel A. The comparison between pre- and postoperative images suggests the preservation of the IFOF after surgery. C: Superior view of the left preoperative OR result from another patient. D: Superior view of the postoperative OR from the patient shown in panel $C$. Tractography suggests the preservation of the OR after surgery in this case. In the inferior right corner of each image, the letters A, P, S, I, L, and R represent directional markers for anterior, posterior, superior, inferior, left, and right, respectively. Figure is available in color online only.

pocampus. Significantly better postoperative seizure control was achieved in group I compared to group II (95\% vs $72 \%$ of Engel class I, respectively). Abel et al. also evaluated the role of the TP in the context of TLE networks using dense electrocorticography. They enrolled 10 consecutive patients who would eventually undergo cortico-amygdalohippocampectomy. Seven patients (70\%) showed TP involvement at seizure onset (group A), while 7 patients showed interictal discharges from TP, and 1 demonstrated early spread to the TP. Of those 7 patients in whom the TP was involved in seizure-onset, only 2 showed TP MRI abnormalities, whereas interictal PET hypometabolism was observed in all patients. ${ }^{25}$

Invasive monitoring is not a routine method of preoperative investigation in many centers. ${ }^{17,21,26}$ Because the participation of the TP in primary seizure organization has been increasingly shown to be a common finding among neurosurgical services, we decided to evaluate the results following resection of mesial structures plus the TP for patients with HS in the context of a noninvasive preoperative investigation. With regards to seizure control, our patients showed a rewarding Engel classification outcome: nearly 75\% achieved Engel class I outcome, and 91.8\% of our patients reached Engel class I or II. Temporal epilepsy surgical approaches, whether selective or not, have shown Engel class I outcomes varying between $62.2 \%$ and $80.4 \%$ for MTLE patients with HS after 2 years of followup. ${ }^{17,27-29}$ Of note, the Engel classification is dependent on the extent of the follow-up, the duration of epilepsy, and the presence of dual and extratemporal pathology, among other factors. ${ }^{18,23,30}$ Although a fair comparison with the current literature data cannot be made, the present study suggested that TP-AH provided similar results to historical TLE series.

\section{TS Analyses}

The most important TS white matter fiber tracts from anterior to posterior are the uncinate fasciculus (UF), IFOF, and OR. Because the UF connects the TP to frontal lobe structures, resection of the anterior temporal lobe inevitably results in disruption of this tract. ${ }^{31}$ Due to this anatomical relationship, our study focused on the preservation 
TABLE 3. Comparisons of neuropsychological variables

\begin{tabular}{lrcc}
\hline \multicolumn{1}{c}{ Variable } & \multicolumn{1}{c}{ Preop $^{*}$} & Postop* $^{*}$ & p Value $\dagger$ \\
\hline Memory & & & \\
\hline RAVLT delayed recall & $-1.50 \pm 0.26$ & $-1.58 \pm 0.33$ & 0.75 \\
\hline RAVLT codification & $-0.88 \pm 0.22$ & $-1.15 \pm 0.28$ & 0.34 \\
\hline RAVLT recognition & $-2.7 \pm 0.65$ & $-5.41 \pm 1.22$ & 0.02 \\
\hline WMS-R verbal memory & $-1.3 \pm 0.16$ & $-2.06 \pm 0.19$ & 0.007 \\
\hline WMS-R visual memory & $-1.6 \pm 0.22$ & $-0.71 \pm 0.22$ & 0.007 \\
\hline WMS-R delayed retrieval & $-1.68 \pm 0.16$ & $-1.99 \pm 0.19$ & 0.14 \\
\hline IQ assessment & & & \\
\hline WAIS-R & $77.1 \pm 14.1$ & $88.5 \pm 9.8$ & $<0.001$ \\
\hline Executive function & & & \\
\hline BNT & $-1.42 \pm 2.29$ & $-0.57 \pm 1.51$ & 0.06 \\
\hline Semantic fluency & $0.42 \pm 1.02$ & $0.15 \pm 1.03$ & 0.72 \\
\hline Phonemic fluency & $-1.4 \pm 0.86$ & $-1.22 \pm 1.5$ & 0.72 \\
\hline
\end{tabular}

* Data given as mean \pm SD; means are adjusted to the time interval between surgery and postoperative neuropsychological assessment.

$\dagger$ False discovery rate corrected $p$ values.

of the more posterior components of the TS. The IFOF is a long association tract that runs immediately rostral to the UF. ${ }^{32}$ According to anatomical postmortem dissections performed by Martino et al., the IFOF occupies the posterior two-thirds of the TS. At the level of the inferior limiting sulcus of the insula, the anteroposterior distance between the limen insula and the anterior edge of the IFOF is shorter on the right side (average $10.4 \mathrm{~mm}$, range 9-12 $\mathrm{mm}$ ) when compared with the left side (average $11.4 \mathrm{~mm}$, range $8-15 \mathrm{~mm}){ }^{33}$ The close proximity between the IFOF and limen insula reinforces the need to respect the limen insula as the posterior limit of TP resection. As suggested by our results, this level of surgical detail is important for preserving the IFOF, which might be only $8-9 \mathrm{~mm}$ from this surgical limit.

The OR is situated in the more posterior portions of the TS. Anatomical postmortem dissections have reported that the more anterior aspect of the OR is located an average of $25 \mathrm{~mm}$ (range $22-30 \mathrm{~mm}$ ) posterior to the temporal lobe anterior aspect, extending $5 \mathrm{~mm}$ (range 3-6 mm) anterior to the hippocampus head and $2 \mathrm{~mm}$ (range 2-3 $\mathrm{mm}$ ) anterior to the anterior tip of the temporal horn. ${ }^{34}$ Siderelated asymmetries have also been reported in anatomical studies of the OR, and the distance between the temporal lobe anterior aspect and Meyer's loop is a predictor for VFD following epilepsy surgery. ${ }^{35,36}$ Imaging studies have revealed that the distance between anterior aspect of the temporal lobe and the Meyer's loop is on average $25 \mathrm{~mm}$ (range 19.9-28.9 mm) in the left hemisphere and $26.6 \mathrm{~mm}$ (range $23.1-29.8 \mathrm{~mm}$ ) in the right hemisphere. ${ }^{36}$ Therefore, left-sided resections carry a higher risk of visual field loss. ${ }^{35}$

Surgical axonal transections are expected to reduce FA and increase MD. Wallerian degeneration due to surgery also consists of two phases of white matter alterations: 1) an acute phase characterized by fragmentation and dying back of axons, associated with $\mathrm{AD}$ reduction; and 2) a chronic phase characterized by phagocytosis, myelin sheath loss, and consequential RD increase. ${ }^{12}$ Regardless of the side of surgery, there were no significant changes in the IFOF and OR tractography following TP-AH, suggesting that respecting the limen insula as the posterior limit tends to anatomically preserve the TS.

\section{Visual Field Analyses}

We observed a postoperative VFD rate of $32.5 \%$, which suggests that using the limen insula as a TS landmark was not sufficient to preserve the OR completely. While this could be due to the anatomical relationship between the Meyer's loop and the anterior tip of the temporal horn, ${ }^{34}$ this VFD rate was nevertheless similar to that recorded from patients who were submitted to other TLE surgical approaches (21\%-58\%). ${ }^{37}$ Although there was an OR postoperative fiber reduction, our DTI data were not sufficiently sensitive to detect VFD attributable to surgery, perhaps because the VFDs were mostly incomplete quadrant defects.

\section{Neurocognitive Assessment}

Our patients demonstrated a significant decline in verbal memory, which was also observed after selective and nonselective approaches in our previous series, and can be related to the limbic system functional disconnection. ${ }^{13} \mathrm{In}$ contrast, the rewarding outcomes after TP-AH (good seizure control, IQ and visual memory improvement, BNT and executive function preservation, acceptable VFD rate) counterbalance the verbal memory decline observed postoperatively. It is also worth noting that the resection of the TP neocortex did not influence the postoperative executive performance or the IQ negatively.

The IFOF is responsible for semantic language processing, as a component of the ventral pathway of the language. ${ }^{33,38}$ Intraoperative electrostimulation of the IFOF elicits semantic paraphasia, ${ }^{33,38}$ and an increased FA in the IFOF is associated with better neurocognitive performance in semantic fluency, BNT, and vocabulary tasks. ${ }^{39}$ Our results revealed preservation of the IFOF, which is compatible with semantic fluency and BNT preservation after surgery. Therefore, defining the limen insula as a posterior limit to TP resection can be an important surgical landmark to ensure anatomical and functional preservation of the IFOF.

\section{Transient Postoperative Complications}

The largest series of patients who underwent amygdalohippocampectomy to treat MTLE reveals similar morbidity among different surgical approaches $(0 \%-10 \%)$. Wieser et al. reported a morbidity rate of $5.73 \%$ in 453 patients (7 patients with hemiparesis and 4 with oculomotor nerve palsy). ${ }^{40}$ Wiebe et al. reported a $10 \%$ morbidity rate with ATL, and Sindou et al. reported a 19\% morbidity rate (meningitis, hemiparesis, hydrocephalus, third cranial nerve palsy), for patients who underwent transsylvian amygdalohippocampectomy. ${ }^{41,42}$

As there was no mortality or permanent morbidity in our study, other than the expected VFD (30\% with quadrant field defect and $2.5 \%$ with hemifield defect), our re- 
sults were within acceptable parameters. In the present series, 6 patients $(9.8 \%)$ had some surgical morbidity, which we classified as minor morbidity and which resolved in 3 months or less. ${ }^{43}$ This percentage is similar to previous reports, and is possibly overestimated due to the small number of patients in our group and our very detailed inclusion of all postoperative findings. If we exclude the findings of aseptic meningitis and CSF under the scalp, there are only 4 patients $(6.5 \%)$ who experienced minor morbidity. These results support an inference that TP-AH has a morbidity rate similar to other surgical approaches for amygdalohippocampectomy.

\section{Limitations}

There are important limitations underlying the conclusions set forth in the present study. Preoperative perimetry was not performed, which may have resulted in a higher estimate of the VFD rate attributable to surgery. Additionally, not all patients underwent postoperative perimetry and neuropsychological evaluation.

\section{Conclusions}

TP-AH showed a seizure control rate that is comparable to historical TLE surgical approaches. Our modified technique demonstrated a tendency of anatomical preservation of the TS, with VFDs comparable to series using selective approaches. Our results suggested that the preservation of the IFOF likely enabled preserved semantic fluency, and that the resection of the TP did not prejudice executive function. TP-AH is a natural evolution of the traditional transsylvian approach for our group, and we believe that TP-AH can be a valuable alternative surgical technique for those groups who perform the transsylvian approach and are observing TP atrophy and TS damage.

\section{Acknowledgments}

This work was funded by the São Paulo Research Foundation (FAPESP), grant number 2013/07559-3.

\section{References}

1. Lee SK. Treatment strategy for the patient with hippocampal sclerosis who failed to the first antiepileptic drug. J Epilepsy Res. 2014;4(1):1-6.

2. Yasuda CL, Tedeschi H, Oliveira ELP, et al. Comparison of short-term outcome between surgical and clinical treatment in temporal lobe epilepsy: a prospective study. Seizure. 2006;15(1):35-40.

3, Yasargil MG, Teddy PJ, Roth P. Selective Amygdalo-Hippocampectomy Operative Anatomy and Surgical Technique. Springer; 1985:93-123.

4. Giacomini L, de Souza JPSA, Formentin C, et al. Temporal lobe structural evaluation after transsylvian selective amygdalohippocampectomy. Neurosurg Focus. 2020;48(4):E14.

5. Hu W-H, Zhang C, Zhang K, et al. Selective amygdalohippocampectomy versus anterior temporal lobectomy in the management of mesial temporal lobe epilepsy: a meta-analysis of comparative studies. J Neurosurg. 2013;119(5):1089-1097.

6. Jain P, Tomlinson G, Snead C, et al. Systematic review and network meta-analysis of resective surgery for mesial temporal lobe epilepsy. J Neurol Neurosurg Psychiatry. 2018;89(11):1138-1144.

7. Josephson CB, Dykeman J, Fiest KM, et al. Systematic re- view and meta-analysis of standard vs selective temporal lobe epilepsy surgery. Neurology. 2013;80(18):1669-1676.

8. Kuang Y, Yang T, Gu J, et al. Comparison of therapeutic effects between selective amygdalohippocampectomy and anterior temporal lobectomy for the treatment of temporal lobe epilepsy: a meta-analysis. Br J Neurosurg. 2014;28(3):374-377.

9. Alvim MKM, Morita ME, Yasuda CL, et al. Is inpatient ictal video-electroencephalographic monitoring mandatory in mesial temporal lobe epilepsy with unilateral hippocampal sclerosis? A prospective study. Epilepsia. 2018;59(2):410-419.

10. Ghizoni E, Almeida JP, Joaquim AF, et al. Modified anterior temporal lobectomy: anatomical landmarks and operative technique. J Neurol Surg A Cent Eur Neurosurg. 2015;76(5):407-414.

11. Blümcke I, Thom M, Aronica E, et al. International consensus classification of hippocampal sclerosis in temporal lobe epilepsy: a Task Force report from the ILAE Commission on Diagnostic Methods. Epilepsia. 2013;54(7):1315-1329.

12. de Souza JPSAS, Ayub G, Pereira PC, et al. Fractional anisotropy of the optic radiations correlates with the visual field after epilepsy surgery. Neuroradiology. 2019;61(12):14251436.

13. Fernandes DA, Yasuda CL, Lopes TM, et al. Long-term postoperative atrophy of contralateral hippocampus and cognitive function in unilateral refractory MTLE with unilateral hippocampal sclerosis. Epilepsy Behav. 2014;36:108-114.

14. Bonner MF, Price AR. Where is the anterior temporal lobe and what does it do? J Neurosci. 2013;33(10):4213-4215.

15. Lambon Ralph MA, Cipolotti L, Manes F, Patterson K. Taking both sides: do unilateral anterior temporal lobe lesions disrupt semantic memory? Brain. 2010;133(11):3243-3255.

16. Tsapkini K, Frangakis CE, Hillis AE. The function of the left anterior temporal pole: evidence from acute stroke and infarct volume. Brain. 2011;134(Pt 10):3094-3105.

17. Elliott CA, Broad A, Narvacan K, et al. Seizure outcome in pediatric medically refractory temporal lobe epilepsy surgery: selective amygdalohippocampectomy versus anterior temporal lobectomy. J Neurosurg Pediatr. 2018;22(3):276282.

18. Dorfer C, Czech T, Aull-Watschinger S, et al. Mesial temporal lobe epilepsy: long-term seizure outcome of patients primarily treated with transsylvian selective amygdalohippocampectomy. J Neurosurg. 2018;129(1):174-181.

19. Schmeiser B, Wagner K, Schulze-Bonhage A, et al. Transsylvian selective amygdalohippocampectomy for mesiotemporal epilepsy: experience with 162 procedures. Neurosurgery. 2017;80(3):454-464.

20. Yaşargil MG, Krayenbühl N, Roth P, et al. The selective amygdalohippocampectomy for intractable temporal limbic seizures. J Neurosurg. 2010;112(1):168-185.

21. Ghizoni E, Matias RN, Lieber S, et al. Clinical and imaging evaluation of transuncus selective amygdalohippocampectomy. World Neurosurg. 2017;100:665-674.

22. Renowden SA, Matkovic Z, Adams CB, et al. Selective amygdalohippocampectomy for hippocampal sclerosis: postoperative MR appearance. AJNR Am J Neuroradiol. 1995;16(9):1855-1861.

23. Barba C, Rheims S, Minotti L, et al. Temporal plus epilepsy is a major determinant of temporal lobe surgery failures. Brain. 2016;139(Pt 2):444-451.

24. Chabardès S, Kahane P, Minotti L, et al. The temporopolar cortex plays a pivotal role in temporal lobe seizures. Brain. 2005;128(Pt 8):1818-1831.

25. Abel TJ, Woodroffe RW, Nourski KV, et al. Role of the temporal pole in temporal lobe epilepsy seizure networks: an intracranial electrode investigation. J Neurosurg. 2018;129(1):165-173.

26. Diehl B, Lüders HO. Temporal lobe epilepsy: when are invasive recordings needed? Epilepsia. 2000;41(suppl 3):S61-S74. 
27. McIntosh AM, Kalnins RM, Mitchell LA, et al. Temporal lobectomy: long-term seizure outcome, late recurrence and risks for seizure recurrence. Brain. 2004;127(Pt 9):20182030.

28. Moura LMVR, Eskandar EN, Hassan M, et al. Anterior temporal lobectomy for older adults with mesial temporal sclerosis. Epilepsy Res. 2016;127:358-365.

29. Sagher O, Thawani JP, Etame AB, Gomez-Hassan DM. Seizure outcomes and mesial resection volumes following selective amygdalohippocampectomy and temporal lobectomy. Neurosurg Focus. 2012;32(3):E8.

30. Jehi L, Yardi R, Chagin K, et al. Development and validation of nomograms to provide individualised predictions of seizure outcomes after epilepsy surgery: a retrospective analysis. Lancet Neurol. 2015;14(3):283-290.

31. Hau J, Sarubbo S, Perchey G, et al. Cortical terminations of the inferior fronto-occipital and uncinate fasciculi: anatomical stem-based virtual dissection. Front Neuroanat. 2016;10:58.

32. Peltier J, Verclytte S, Delmaire C, et al. Microsurgical anatomy of the temporal stem: clinical relevance and correlations with diffusion tensor imaging fiber tracking. J Neurosurg. 2010;112(5):1033-1038.

33. Martino J, Vergani F, Robles SG, Duffau H. New insights into the anatomic dissection of the temporal stem with special emphasis on the inferior fronto-occipital fasciculus: implications in surgical approach to left mesiotemporal and temporoinsular structures. Neurosurgery. 2010;66(3)(Suppl Operative):4-12.

34. Rubino PA, Rhoton AL Jr, Tong X, Oliveira Ed. Three-dimensional relationships of the optic radiation. Neurosurgery. 2005;57(4)(suppl):219-227.

35. Dreessen de Gervai P, Sboto-Frankenstein UN, Bolster RB, et al. Tractography of Meyer's Loop asymmetries. Epilepsy Res. 2014;108(5):872-882.

36. Yogarajah M, Focke NK, Bonelli S, et al. Defining Meyer's loop-temporal lobe resections, visual field deficits and diffusion tensor tractography. Brain. 2009;132(Pt 6):1656-1668.

37. Schmeiser B, Daniel M, Kogias E, et al. Visual field defects following different resective procedures for mesiotemporal lobe epilepsy. Epilepsy Behav. 2017;76:39-45.

38. Duffau H, Thiebaut de Schotten M, Mandonnet E. White matter functional connectivity as an additional landmark for dominant temporal lobectomy. J Neurol Neurosurg Psychiatry. 2008;79(5):492-495.

39. Houston J, Allendorfer J, Nenert R, et al. White matter language pathways and language performance in healthy adults across ages. Front Neurosci. 2019;13:1185.
40. Wieser HG, Ortega M, Friedman A, Yonekawa Y. Long-term seizure outcomes following amygdalohippocampectomy. $J$ Neurosurg. 2003;98(4):751-763.

41. Sindou M, Guenot M, Isnard J, et al. Temporo-mesial epilepsy surgery: outcome and complications in 100 consecutive adult patients. Acta Neurochir (Wien). 2006;148(1):39-45.

42. Wiebe S, Blume WT, Girvin JP, Eliasziw M. A randomized, controlled trial of surgery for temporal-lobe epilepsy. $N$ Engl J Med. 2001;345(5):311-318.

43. Rydenhag B, Silander HC. Complications of epilepsy surgery after 654 procedures in Sweden, September 1990-1995: a multicenter study based on the Swedish National Epilepsy Surgery Register. Neurosurgery. 2001;49(1):51-57.

\section{Disclosures}

The authors report no conflict of interest concerning the materials or methods used in this study or the findings specified in this paper.

\section{Author Contributions}

Conception and design: de Souza, Ayub, Ghizoni. Acquisition of data: de Souza, Ayub, Nogueira, Zanao, Lopes, Domene, Marquez, Ribeiro, Rogerio. Analysis and interpretation of data: de Souza, Ayub, Nogueira, Zanao, Pimentel-Silva, Vasconcellos, Ghizoni. Drafting the article: de Souza, Ayub, Ghizoni. Critically revising the article: de Souza, Ayub, Zanao, Yasuda, Campos, Rogerio, Joaquim, Cendes, Tedeschi, Ghizoni. Reviewed submitted version of manuscript: de Souza, Nogueira. Approved the final version of the manuscript on behalf of all authors: de Souza. Statistical analysis: Pimentel-Silva. Study supervision: Cendes, Ghizoni.

\section{Supplemental Information}

Online-Only Content

Supplemental material is available with the online version of the article.

Supplemental Materials. https://thejns.org/doi/suppl/10.3171/ 2020.3.JNS192624.

\section{Correspondence}

João Paulo Sant Ana Santos de Souza: University of Campinas, São Paulo, Brazil. souza.medicine@gmail.com. 